\title{
Optimal Environmental Taxation and Enforcement Policy*
}

\author{
Philippe Bontems ${ }^{\dagger} \quad$ Jean-Marc Bourgeon ${ }^{\ddagger}$
}

\begin{abstract}
We study the optimal environmental taxation and enforcement policy when (i) the regulator does not know the firms' abatement costs, (ii) penalties for tax evasion are limited, and (iii) monitoring of pollution is costly. We show that the threat of being audited alter the usual firms' incentives to over-estimate their abatement costs. In particular, depending on the firms' abatement costs, the optimal policy may involve over or under-deterrence compared to the full information outcome. We then investigate the properties of a pollution standard. We show that this policy comes close to an environmental tax once the economic incentives of the accompanying enforcement policy are considered.
\end{abstract}

Key-words : Environmental Taxation, Law Enforcement, Tax Evasion, Adverse Selection.

JEL Classification : D62, D82, H21, H26, H32

*Corresponding author: Philippe Bontems, Address : INRA, Manufacture des Tabacs, Bât F., 31000 Toulouse, France. Fax : (33) 5611285 20. Email : bontems@toulouse.inra.fr. Financial support from PIREE program (Ministère de l'Environnement et de l'Aménagement du Territoire) is gratefully acknowledged. We thank discussants and participants at the following conferences and seminars: University of Quebec at Montreal, CREE 2000 (Guelph U.), PIREE conference (Nice), EAERE 2001 (Southampton), ESEM 2001 (Lausanne). Finally, we thank the Editor and the two anonymous referees for their helpful comments.

${ }^{\dagger}$ University of Toulouse (INRA, IDEI).

‡THEMA, Université de Paris-X Nanterre. 


\section{Introduction}

Environmental taxes are an important part of the system for regulating pollution in several European countries. At member state level, there has been a continuing increase in the use of environmental taxes over the last decade, especially in Scandinavia, Austria, Belgium, France, The Netherlands, Germany and The United Kingdom (European Environmental Agency, 1996). It appears from textbook discussions that the first major advantage of environmental taxes is to provide polluters with the correct incentives to internalize external costs. This leads to a better integration of economic

and environmental policies. Second, such taxes produce revenue which may be used to improve environmental expenditures and/or to reduce the distortions due to taxation on labor, capital and savings (the so-called double dividend).

However, a careful design of environmental taxes should also include an enforcement mechanism to deter non compliance and tax evasion. Monitoring and enforcement may not be the first elements that come to mind in this field and indeed, these issues are often ignored by both academic and policy makers when discussing environmental policy reform (Cohen, 1999). This general lack of attention may have negative consequences for environmental quality and for social welfare. Trying to implement stricter regulations than the existing ones may result in increased pollution levels if the agency cannot control the firms activities and enforce compliance. Moreover, ignoring monitoring and enforcement costs in the case of a new regulation might lead the public authorities to implement costlier policies than the current ones. Consequently, when investigating the optimal regulation of polluting firms, the analysis must include the facts that the monitoring of actual polluters' emissions is costly and, more generally, that the information needed by the regulator is decentralized in the economy (see Lewis, 1996, for a survey on these issues). In particular, if it is realistic to assume that the regulator possesses aggregate information on polluters' abatement costs, it seems doubtful that 
she can observe the costs of each individual firm. Moreover, many systems for enforcing pollution controls are impeded by legislation that puts limitations on the regulator's power to punish non compliance.

We shall then analyze a nonlinear environmental taxation assuming that emission levels can be observed through a costly audit and that abatement costs remain private information even when an audit is performed. Given this information structure, we derive and investigate the properties of the optimal tax and enforcement policy.

Our analysis brings out the following points. i/ The optimal tax is different from the Pigovian level because of the second dividend of the environmental tax and of the social cost of monitoring. Both effects imply greater allowances to polluting firms (under-deterrence). This phenomenon is easily understood: With a strictly positive social cost of public funds, due to imperfections of existing tax systems on labor or capital, the regulator is tempted to raise more money with environmental taxes than of the Pigovian levels. Moreover, costly enforcement per se entails pollution levels strictly higher than the Pigovian levels in order to reduce the incentive to evade. ii/ The usual rule of equalizing private marginal benefits to social marginal damage is violated further, because of complex distortions due to the adverse selection problem which give rise to firms-specific marginal tax rates. Under adverse selection on the abatement technology but without monitoring cost, Baron (1985) and Laffont (1994) show that the optimal tax schedule entails lower emission levels the higher the firm's abatement costs (over-deterrence effect due to the adverse selection problem). With costly enforcement and self-reporting, we show that the agency has to take account of another adverse-selection effect: under-deterrence increases the informational rents of profitable firms but decreases the inspection effort on less efficient firms. The regulator has thus to arbitrate between two effects when designing the taxation policy: an increase in the emission levels for a given firm allows the reduction in the inspection efforts on less profitable firms but causes a decrease in the tax collection on more profitable ones. iii/ 
Since the optimal tax policy is intimately related to the monitoring capabilities of the agency, monitoring and tax policies must be designed jointly. In particular, optimal monitoring efforts of the agency are inversely related to the amounts of tax paid by the firms (and thus, to their emission levels). iv/ Command-and-control instruments are closer to economic instruments once the economic incentives of their enforcement policies are considered. We investigate the properties of a pollution standard, where the firms are allowed to pollute up to a given level (the emission standard) by paying a lump-sum transfer (the license fee) and where the agency verifies the firms' compliance with a uniform inspection probability. We show that the optimal standard policy induces the less efficient firms to pollute at the standard in accordance with their licence. Consequently these firms are not fined when inspected. However, more profitable firms pollute over the standard and pay the corresponding penalty when inspected. Consequently, this licence cum-fine schedule resembles a taxation scheme. Malik (1992) already observed that an environmental standard policy may be considered as a special case of taxation policy. A standard policy appears to be a restricted case of a taxation policy because of the "marginal deterrence" induced by the penalty schedule of its enforcement policy. Using an increasing fine schedule allows the agency to introduce efficiency considerations in an otherwise rather inefficient policy.

Although there has been rapid growth in theoretical and empirical studies on enforcement of environmental policies over the last years (see Cohen, 1999, for a recent survey), only few papers have analyzed the case of environmental taxes. Swierzbinski (1994) analyses the optimal regulation when abatement costs are private information and when monitoring is costly. While assuming the regulator is allowed to reward compliant firms, Swierzbinski shows that the optimal mechanism resembles a depositrefund system and that the abatement schedule is affected downward by both adverse selection and monitoring problems. This result is mainly due to the policy objectives he considers. In his framework, the environmental agency wants firms to allocate funds 
to abating polluting emissions up to a maximum amount, which corresponds to the firms' opting out cost. In particular, with a retirement cost equal to zero, the agency has to subsidize firms to induce abatement. We encountered the opposite situation in the case of environmental taxation we investigated. We show that the perfect information schedule is diversely affected by adverse selection and monitoring problems. This framework allows us to detail these effects thoroughly, especially the adverse selection effect in association with the monitoring policy. It also enables us to give a complete characterization of the optimal emission schedule. ${ }^{1}$

In their study on marginal deterrence, Mookherjee and Png (1994) analyze the optimal enforcement of a standard. Our analysis extends this model by considering the possibility of raising money through a licence fee paid by every active firm. Since they take the opposite viewpoint that fines are socially costly, it comes as no surprise that their results differ from ours. However, we show that it is socially efficient to associate a licence fee with the standard even in the case of negligible second-dividend aspects. Firms extracting very low benefits from emissions choose not to pollute because the benefit they derive from polluting up to the standard is smaller than the fee they have to pay. Moreover, we show that profitable firms are divided into two (non empty) subsets. Medium-profitability firms decide to pay the fee and emit up to the standard. Even for these firms the standard exerts a real constraint. Only relatively high-profitability firms exceed the standard. We also analyze the adverse-selection problem generated by the second-dividend aspect in this context. The imitation problem has a downward effect on the optimal emission schedule for firms exceeding the standard. While Mookherjee and Png conclude that the marginal expected penalty should be strictly less than

\footnotetext{
${ }^{1}$ Swiersbinsky also considers the stick-and-carrot policy of penalties-cum-tax-rebates. For deterrence purposes, and given that audits do not reveal the firms' abatement costs, tax-rebates and penalties are symmetric. To enforce risk-neutral firm's compliance, only the payment expected in case of fraud matters, i.e. total penalty amount minus tax-rebate times the probability of an audit. The larger the payment, the fewer the inspections. In our analysis, we do not allow for tax-rebates to stick to actual environmental taxation policies.
} 
the corresponding marginal social harm, we show that when second-dividend aspects matter, and depending on the efficiency of the firm, the marginal expected penalty for violating the standard can be higher or lower than the marginal damage.

Applications of principal-agent models with audit have been developed in various fields like insurance, income taxation or monopoly regulation. The key differences between our problem and these models are that they usually assume that the maximal penalty depends on the private information of the agents and/or that the regulator can reward honesty. As a result, the incentive constraints of audit models are badly behaved. This fact was first recognized by Baron and Besanko (1984) in their analysis of monopoly regulation. For example, in most studies on income taxation, the labor supply is assumed to be given and auditing the income allows access to the entire agent's private information. The maximum fine can thus be set to the entire benefit the agent extracts from evading, which is type-dependent (see Border and Sobel, 1987 and Chander and Wilde, 1998). ${ }^{2}$ However, in our problem, the audit does not reveal the firm's benefit, which implies that the maximum penalty cannot be type-dependent. Moreover, as it is often the case in practice, we forbid tax rebates in case of compliance. Using standards arguments, we show that the optimal fines are not type-dependent: the fine is either 0 , if the firm does not evade, or equal to the maximal penalty otherwise. This simple penalty scheme allows to handle the two aspects of the audit problem separately: The inspection/penalty schedule is intended to solve the evasion problem only, and the tax/emissions schedule to solve the mimicking problem. Once it is verified that no firm is induced to evade, the incentive constraints of the remaining adverseselection problem are well-behaved.

The paper proceeds as follows. Section 2 is devoted to notation, assumptions and the derivation of the optimal policy in the perfect information benchmark case. Section

\footnotetext{
${ }^{2}$ See Cremer and Gahvari (1996) for a model with endogeneous labor supply where the agent's income is the result of two unobservable variables, ability and labor supply, the latter being discovered through a costly audit.
} 
3 offers the main results concerning the optimal taxation and enforcement policy. In section 4, we study the optimal enforcement of an environmental standard. The last section comes to a conclusion. Most proofs are in the appendix.

\section{The model}

Consider an economy consisting of a continuum of firms with mass unity. Firms differ in a one-dimensional measure of their private benefit of pollution. Profitability parameter $\theta$ is distributed over a non-negative interval $\Theta=[\underline{\theta}, \bar{\theta}]$, according to distribution function $G$ and probability density function $g \equiv G^{\prime}$ with $g(\theta)>0$ for all $\theta \in \Theta$. Each firm chooses an emission level $q$ (or abatement effort) which yields the profit $\pi(q, \theta)$ and we normalize the set $\Theta$ by assuming that $\partial_{\theta} \pi>0 .{ }^{3}$ Without regulation, individual emission level $q^{\circ}(\theta)$ satisfies

$$
q^{\circ}(\theta) \in \arg \max _{q} \pi(q, \theta)
$$

We assume that $\partial_{q q} \pi(q, \theta) \leq 0$ and $\partial_{q \theta} \pi(q, \theta)>0$ for all $q<q^{\circ}(\theta)$, i.e., that the marginal benefit of pollution decreases with pollution and increases with the firms' type. This implies that emission pattern $q^{\circ}(\cdot)$ is a non-decreasing function of $\theta$. The latter assumption corresponds to the well-known Spence-Mirrlees single crossing condition that simplifies the analysis of the adverse-selection problems. ${ }^{4}$ The profit of the type- $\theta$ firm without regulation is given by

$$
\pi^{\circ}(\theta) \equiv \pi\left(q^{\circ}(\theta), \theta\right)
$$

which is an increasing function of $\theta$ under the assumption $\partial_{\theta} \pi>0$.

Environmental damage $D$ is supposed to depend on aggregate pollution $Q=$ $\int_{\underline{\theta}}^{\bar{\theta}} q(\theta) g(\theta) d \theta$ according to the relation $D=Q d$ where $d$ is the marginal environ-

\footnotetext{
${ }^{3}$ We denote by $\partial_{x} f$ the partial derivative of a function $f(\cdot)$ with respect to the variable $x$.

${ }^{4}$ We also briefly analyze the case where $\partial_{q \theta} \pi(q, \theta)<0$, i.e., where more profitable firms use greener technologies and pollute less.
} 
mental damage. ${ }^{5}$ The regulator's task is thus to design an environmental tax schedule that induces producers to internalize this damage. ${ }^{6}$ More precisely, we assume that the regulator's objective is to maximize the expected social welfare given by

$$
\int_{\underline{\theta}}^{\bar{\theta}}\{\pi(q(\theta), \theta)-d q(\theta)+\lambda t(\theta)\} g(\theta) d \theta
$$

where $\lambda \geq 0$ and $t(\theta)$ is the tax paid by the type- $\theta$ firms. The term $\lambda \int_{\underline{\theta}}^{\bar{\theta}} t(\theta) g(\theta) d \theta$ corresponds to the indirect social benefit of an environmental taxation, commonly called the "second dividend": Using tax to correct the externality also allows the government to diminish the tax burden that weighs on the rest of society. This reduction decreases the deadweight losses associated with other existing tax systems, like income taxation, that induce distortions in the economy. $\lambda$ is a per monetary unit measure of these deadweight losses $(1+\lambda$ is commonly called the shadow cost of public funds). Raising $t$ on a polluting firm with an environmental tax allows the government to diminish other taxes by the same amount and thus induces an indirect social gain equal to $\lambda t$. As a consequence, the government wants to raise as much money as possible through an environmental tax in order to save on costlier tax systems, and thus capture the entire profit of the polluting firms. ${ }^{7}$ More precisely, if the regulator was able to observe the firms' emissions directly, it would be possible to implement the perfect information outcome via a tax-emissions schedule $\left\{t^{*}(\theta), q^{*}(\theta), \theta \in \Theta\right\}$ satisfying

$$
\partial_{q} \pi\left(q^{*}(\theta), \theta\right)=d /(1+\lambda)
$$

and

$$
t^{*}(\theta)=\pi\left(q^{*}(\theta), \theta\right)
$$

\footnotetext{
${ }^{5}$ This assumption is made to simplify the algebra while keeping the main insights of our analysis. Extensions to more general damage functions is straightforward.

${ }^{6}$ For simplicity, we do not consider here the impact of the regulation on the consumers' surplus.

${ }^{7}$ We implicitly assume that there is no political constraint that limits the government's latitude in setting up environmental taxation. Such political constraints may be taken into account by requiring that the firms' profits after tax be greater than a given level $\bar{\pi}>0$. By assuming $\bar{\pi}=0$ we save on notation without changing the qualitative results of the analysis.
} 
When $\lambda=0$, the optimal emission pattern corresponds to the first-best levels, defined by the usual rule of equating marginal private benefit $\partial_{q} \pi\left(q^{*}(\theta), \theta\right)$ to marginal social damage $d$. Moreover, there is no reason in that case to charge firms per se. The optimal policy may be implemented with a Pigovian tax equal to the marginal social damage regardless of the private information of firms. This is no longer the case when there are distortions in the rest of the economy $(\lambda>0)$. In that case, the regulator seeks to raise as much tax revenue as possible with the environmental tax and she has not only to consider the environmental objective of the policy but also its incentive aspect. A direct effect of the distortions is that the regulator is led to allow firms to over-pollute compared to first-best levels. This increase in emission levels allows the regulator to raise more revenue, since the entire benefit of pollution is captured by the agency. However, such a capture is possible only when the regulator is able to assess the benefit that each firm extracts from polluting and if the firms are not tempted to evade taxation. Moreover, assuming that the regulator desires to implement a given tax-emissions schedule, it is doubtful that she will be able to check the firms' compliance with the legislation at no cost. More generally, the agency has to take account of the informational aspect of the problem when designing its policy. We assume the following information structure. First, individual profitability is the firm's private information, while the regulator only knows the distribution of types. Pollution is not directly observable by the regulator either, but can be discovered through a costly audit. However, the audit cost increases with the number of firms audited. The agency has thus to balance the benefits of an audit with its cost.

Given this information structure, we derive the optimal revelation mechanism and investigate the properties of optimal tax and audit policies. The process of regulation and inspection is modeled as a three-stage game. First, the regulator chooses a mechanism. Second, the firm reports its type and simultaneously chooses its pollution level. Third, the contract is implemented, that is, the firm is monitored according to 
the probability determined by the type's report. If no audit occurs, the firm pays only the tax according to its announcement. In the case of auditing, it pays the transfer corresponding to its announcement and the result of the audit.

More formally, a mechanism for the environmental agency consists of four functions: $q(\tilde{\theta}), \mu(\tilde{\theta}), t(\tilde{\theta})$ and $f(\tilde{\theta}, q)$, where $\tilde{\theta}$ is the reported type and $q$ the pollution as revealed by the audit. A firm that has reported a profitability parameter $\tilde{\theta}$ is assigned to an emission level $q(\tilde{\theta})$, pays a $\operatorname{tax} t(\tilde{\theta})$ and is audited with probability $\mu(\tilde{\theta})$. In case of auditing, it pays a fine $f(\tilde{\theta}, q) \geq 0$ if $q \neq q(\tilde{\theta}) .^{8}$

A firm may misbehave along two ways. First, it may misreport its type while producing the emission level assigned to the reported type. In this case, the firm perfectly mimics another type but does not evade, and the result of the audit gives $q=q(\tilde{\theta})$. This mimicking cannot be discovered by auditing the firm, because the inspection reveals only the level of pollution and not the firm's type and profit level. Secondly, it may evade by choosing an emission level different from the one it is assigned to by its report, i.e. $q \neq q(\tilde{\theta})$. This shirking is detected by auditing the firm.

As the revelation principle applies in our context, we can restrict the search of the optimal mechanism to the set of direct and incentive-compatible mechanisms without loss of generality. ${ }^{9}$ The expected profit of a type- $\theta$ firm that announces to be of type $\tilde{\theta}$ and that pollutes at level $q$ is given by

$$
U(\theta, \tilde{\theta}, q)=\pi(q, \theta)-t(\tilde{\theta})-\mu(\tilde{\theta}) f(\tilde{\theta}, q)
$$

Compared to the perfect information situation, a positive expected profit corre-

\footnotetext{
${ }^{8}$ Since the fine is constrained to be non negative, we forbid tax rebates. Consequently, in accordance to the general practice, the agency cannot reward firms in case of compliance. See Swierzbinski (1994) for an analysis of the incentive properties of tax rebates.

${ }^{9}$ Thanks to the Taxation Principle, the menu of contracts described above is equivalent to a nonlinear taxation schedule associated with monitoring and penalty policies (see, e.g., Laffont and Tirole, 1993). Indeed, the regulator could ask each firm to report its $\tilde{q}$ pollutions level and pay the corresponding tax $\tau(\tilde{q})$, where $\tau(\cdot)$ is a non linear function of the declared emissions level. The firm is audited with probability $\xi(\tilde{q})$ and pays a penalty $\varphi(\tilde{q}, q)$ when the true pollution level $q$ is different from the reported $\tilde{q}$.
} 
sponds to an informational rent for the firm that enjoys it. Denote by $R(\theta)$ the expected profit of a type- $\theta$ firm that truthfully announces its type and pollutes according to the regulator's requirement. The first type of incentive constraints can be written as

$$
R(\theta) \equiv U(\theta, \theta, q(\theta)) \geq U(\theta, \tilde{\theta}, q(\tilde{\theta}))
$$

for all $\theta$ and $\tilde{\theta}$ in $\Theta$, where $U(\theta, \tilde{\theta}, q(\tilde{\theta}))$ corresponds to the expected profit of a type- $\theta$ firm that perfectly mimics a firm of type $\tilde{\theta}$, i.e., that announces $\tilde{\theta}$, pays the $\operatorname{tax} t(\tilde{\theta})$ and pollutes at $q(\tilde{\theta})$. The constraints (IC1) insure that the firm does not improve its expected profit by choosing the tax-pollution pair designed for another type. The second type of incentive constraints is given by

$$
R(\theta) \geq \max _{\tilde{\theta}, q} U(\theta, \tilde{\theta}, q)
$$

for all $\theta$. The difference between (IC1) and (IC2) is as follows: with the latter, the firm does not constraint itself to mimic another existing type by choosing the pollution level which corresponds to its announcement. Instead, it allows itself to choose any pollution level. If the regulator's mechanism satisfies (IC2), the firm is better off choosing the tax-pollution pair designed for its type rather than produce any other emission level and try to pay any other tax amount. Obviously, if (IC2) constraints are satisfied, so are (IC1). Taking account of (IC2) constraints only is thus sufficient to pursue the analysis. However, it is useful to distinguish between these two sets of constraints, as shown below.

To satisfy (IC2), the regulator must be able to inflict severe punishments to the firm. However, the fines that the regulator may inflict are usually bounded, i.e.

$$
f(\tilde{\theta}, q) \leq \bar{F}
$$

where $\bar{F}$ is the exogenous maximum fine due, for example, to limited firms' liability. ${ }^{10}$ It is straightforward that the maximum fine should be applied to any firm that is

\footnotetext{
${ }^{10}$ This assumption has been largely discussed in the literature and is founded on several justifications
} 
caught shirking. This does not affect (IC1) constraints and relaxes (IC2) constraints. Hence, we have $f(\tilde{\theta}, q)=\bar{F}$ whenever $q \neq q(\tilde{\theta}) .{ }^{11}$ The same reasoning applies if the audit reveals that the firm is compliant with the rule: We can relax (IC2) constraints by setting $f(\tilde{\theta}, q(\tilde{\theta}))=0$ without changing (IC1) constraints. Indeed, with risk neutral firms, all incentives to truthfully report the type can be embedded in tax level $t(\theta) .{ }^{12}$ Consequently, penalty schedule $f(\cdot)$ reads as

$$
f(\theta, q)= \begin{cases}0 & \text { if } q=q(\theta) \\ \bar{F} & \text { otherwise }\end{cases}
$$

We thus have a simple penalty scheme: the payment is either 0 if the firm does not evade, or is equal to the maximal penalty otherwise. This simplicity allows us to separate the two aspects of the audit problems: the evasion problem is taken care of by the inspection-penalty schedule, whereas the inefficient mimicking problem is deterred by the tax-emissions schedule. Indeed, since compliance is not rewarded, the expected profit of a type- $\theta$ firm with an incentive-compatible mechanism becomes

$$
R(\theta)=\pi(q(\theta), \theta)-t(\theta)
$$

which corresponds to the informational rent of a firm when emissions are observable without cost by the principal.

As mentioned above, auditing firms entails a cost that diminishes the expected social welfare (1). This cost, by expanding public expenses, also diminishes the indirect

ranging from the limited liability of shareholders (the extension of firms liability to third parties, such as lenders or contractors, has been recently considered, see Boyer and Laffont, 1997) to the functioning of the judicial system (even if prescribed by laws, courts are usually reluctant to enforce penalties that are not reasonably related to the damage). More technically, unlimited liability gives rise to the improbable result that by using an arbitrarily large penalty in case of fraud, the agency can deter tax evasion at almost no cost (as pointed out by Border and Sobel, 1987). One possibility often investigated in the literature is to assume that maximal penalty is limited by the additional profit the firms can extract from polluting. However, in our context, the audit does not allow the agency to appraise the firms' benefits. The maximum fine has thus to be the same for every firm.

${ }^{11}$ Moreover, we must consider the so-called Becker's conundrum: Given that the inspection effort is socially costly, it is optimal to increase penalties as much as possible and to minimize the probability of costly auditing.

${ }^{12}$ Formally, any scheme $q(\cdot), t(\cdot), \mu(\cdot), f(\cdot)$ with $f(q(\theta), \theta) \geq 0$ may be replaced by a schedule $q(\cdot), \hat{t}(\cdot), \mu(\cdot), \hat{f}(\cdot)$ with $\hat{t}(\theta)=t(\theta)+\mu(\theta) f(q(\theta), \theta)$ and $\hat{f}(q(\theta), \theta)=0$. 
benefit of the environmental taxation. Assuming that the cost of auditing a type- $\theta$ firm with a probability $\mu(\theta)$ is given by $c \mu(\theta)$, the social welfare $(1)$ becomes $^{13}$

$$
W=\int_{\underline{\theta}}^{\bar{\theta}}\{\pi(q(\theta), \theta)-d q(\theta)+\lambda t(\theta)-(1+\lambda) c \mu(\theta)\} g(\theta) d \theta
$$

This objective is constrained by the participation of the firms, i.e.

$$
R(\theta) \geq 0
$$

This insures that the firms' revenues are at least equal to their profits if they choose not to pollute (normalized to 0 , see footnote 7 ). Finally, the audit probability must satisfy

$$
0 \leq \mu(\theta) \leq 1
$$

for all $\theta \in \Theta$. The agency's program may thus be written as

$$
\max _{q(\cdot), t(\cdot), f(\cdot), \mu(\cdot), R(\cdot)} W:(\mathrm{IC} 1),(\mathrm{IC} 2),(\mathrm{IR}),(5),(7)
$$

where $W$ is given by (6), and the right hand sides of (IC1) and (IC2) are deduced from (2) and (4).

\section{Analysis}

Problem I presents five sets of inequality constraints and cannot be solved directly. We shall proceed by presenting intermediate results (lemmas 1 and 2) to transform this general problem into a simple (although parametric) optimal control program (Program III). The following lemma allows us to simplify the incentive and profit constraints.

Lemma 1 Assuming a non-decreasing emission schedule $q(\cdot)$ such that $q^{\circ}(\theta) \geq q(\theta)$ for all $\theta \in \Theta$, the sets of constraints (IC1), (IR) and (IC2) reduce to

$$
\dot{R}(\theta)=\partial_{\theta} \pi(q(\theta), \theta)
$$

\footnotetext{
${ }^{13}$ The assumption of a constant marginal monitoring cost is made for analytical convenience. Extensions to more general cost functions are straightforward.
} 


$$
R(\underline{\theta}) \geq 0
$$

and

$$
R(\bar{\theta}) \geq \pi^{\circ}(\bar{\theta})-K
$$

with

$$
\mu(\theta) \geq(K-\pi(q(\theta), \theta)+R(\theta)) / \bar{F}
$$

and where

$$
K=\min _{\theta}\{t(\theta)+\mu(\theta) \bar{F}\}
$$

Proof. (IR'), (IC1') and the monotonicity constraint on $q(\cdot)$ are derived from standard arguments (see, e.g., Guesnerie and Laffont, 1984). From (9), we can rewrite (IC2) constraints as

$$
K \geq \pi^{\circ}(\theta)-R(\theta)
$$

(IC2') follows from the fact that

$$
\begin{aligned}
\frac{d}{d \theta}\left[\pi^{\circ}(\theta)-R(\theta)\right] & =\partial_{\theta} \pi\left(q^{\circ}(\theta), \theta\right)-\partial_{\theta} \pi(q(\theta), \theta)=\int_{q(\theta)}^{q^{\circ}(\theta)} \partial_{\theta q} \pi(u, \theta) d u \\
& \geq 0
\end{aligned}
$$

since $\partial_{\theta q} \pi \geq 0$ when $q(\theta) \leq q^{\circ}(\theta)$. (9) also implies

$$
\begin{aligned}
K & \leq t(\theta)+\mu(\theta) \bar{F} \\
& =(\pi(q(\theta), \theta)-R(\theta))+\mu(\theta) \bar{F}
\end{aligned}
$$

from (5). Rearranging terms gives (8).

Given (IC1'), in order to deter imitation of low profitability firms by high ones, the informational rents have to be increased according to the (marginal) advantage in term of profitability at the assigned pollution levels. Condition (IR') states that if the firm with the lowest type enjoys positive rent this guarantees that all other (more profitable) firms will. A monotonic emission schedule and conditions (IC1') 
and (IR') are reminiscent of the reduced incentive constraints of standard adverseselection models, as explained in Guesnerie and Laffont (1984). In addition to these no-mimicking conditions, (IC2'), (8) and (9) allow to deter tax evasion. Any shirking firm would choose to pollute at its private optimum $q^{\circ}(\theta)$ and would announce the type that minimizes the sum of the corresponding expected penalty and environmental tax. Since $\pi^{\circ}(\theta)$ increases with $\theta$, the most profitable firms extract the largest profit from such a strategy. Condition (IC2') states that by deterring the most profitable firm from evading it ensues that all other (less profitable) firms will follow the policy requirements. Evasion constraints reduce to (IC2') under the assumption that the agency does not oblige firms to over-pollute compared to their selfish levels, which we assume in the following. ${ }^{14}$ Condition (8) recalls that minimal inspection efforts are necessary to maintain these incentives, whereas (9) defines the minimal expected cost of evading then incurred by shirking firms. Once it is verified that firms are deterred from evading (by designing an audit policy that insures that the most profitable firms will not), the simple fine schedule brings the situation back to the standard problem of designing a contract in a pure adverse-selection setting: The mimicking incentive constraints are the same as those of a pure contract problem with perfect observability of the emission levels, and usual results on second-order conditions of these models hold..$^{15}$

\footnotetext{
${ }^{14}$ It may appear that the regulator finds it profitable to reallocate pollution towards the most efficient firms so that they over-pollute compared to their status-quo levels (see corollary 4). However, allowing for over-pollution at the optimum could result in evasion constraints binding for interior firms' types, involving more complicated algebra, as explained in Jullien (2000).

${ }^{15}$ However, to obtain a monotonic emission schedule conditions are more stringent than in the case of free observability of emissions as explained below.
} 
After substituting (5) for $t(\cdot)$, Lemma 1 allows us to transform program I to

$$
\begin{aligned}
& \max _{q(\cdot), \mu(\cdot), R(\cdot), K} \int_{\underline{\theta}}^{\bar{\theta}}\{(1+\lambda)(\pi(q(\theta), \theta)-c \mu(\theta))-d q(\theta)-\lambda R(\theta)\} g(\theta) d \theta \\
& \text { s.t. } \\
& \dot{R}(\theta)=\partial_{\theta} \pi(q(\theta), \theta) \\
& R(\bar{\theta}) \geq \pi^{\circ}(\bar{\theta})-K \\
& R(\underline{\theta}) \geq 0 \\
& \mu(\theta) \geq(K+R(\theta)-\pi(q(\theta), \theta)) / \bar{F} \\
& 0 \leq \mu(\theta) \leq 1
\end{aligned}
$$

where the monotonicity condition on the emission schedule $q(\cdot)$ and conditions $0 \leq$ $q(\cdot) \leq q^{\circ}(\cdot)$ are neglected. We thus have to verify that these conditions hold with the emission scheme solution of program II.

This program may be simplified further by observing that constraints (IR') and (8) are binding at the optimum, as stated formally in the following lemma.

Lemma 2 At the optimum of program II we have

(i) $R(\underline{\theta})=0$

(ii) $\mu(\theta)=(K+R(\theta)-\pi(q(\theta), \theta)) / \bar{F}$ for all $\theta \in \Theta$.

(iii) $\mu(\theta)>0$ whenever $q(\theta)<q^{\circ}(\theta)$

Proof. (i). Assume $R(\underline{\theta})>0$ at the optimum. Using (IC1'), we have

$$
R(\theta)=R(\underline{\theta})+\int_{\underline{\theta}}^{\theta} \partial_{\theta} \pi(q(u), u) d u .
$$

Since $R(\cdot)$ affects the program's objective negatively, reducing $R(\underline{\theta})$ increases the objective while satisfying the constraints, hence a contradiction. (ii). Assume that (8) is slack on a non-degenerate subset $\Theta$ at the optimum. We may reduce $\mu(\theta)$ slightly on this subset and still satisfy (8). This diminishes audit cost and thus increases the objective, hence a contradiction. (iii). We have

$$
K+R(\theta)-\pi(q(\theta), \theta) \geq K+R(\theta)-\pi^{\circ}(\theta) \geq 0
$$


where the last inequality comes from (IC2). So, unless $q(\theta)=q^{\circ}(\theta)$, we have $\mu(\theta)>0$.

By (i), the tax collected on the lowest profitable firm corresponds to the entire benefit it obtains from polluting. Since informational rents are increasing, the other (more profitable) firms benefit from the asymmetric information. As stated point (ii), at the optimum, the expected cost of evading is the same whatever the announcement, given by $K=t(\theta)+\mu(\theta) \bar{F}$ for all $\theta$. Since this cost is constant, the optimal tax schedule and inspection rate are negatively related: The lower the tax paid by the firm, the higher the probability it will be inspected. Since $q(\cdot)$ and $t(\cdot)$ are non-decreasing, the inspection rate is thus a non-increasing function of the firm's type. Consequently, the more a firm pollutes, the less likely it is to be inspected. This can be easily understood: One of the agency's tasks is to deter firms from cheating about their pollution levels. It is tempting for a firm to evade by announcing a low emission level. To deter such shirking, the agency has thus to increase the probability of inspection for firms paying low taxes. Finally, by (iii), the agency has to check compliance if it wants to induce lower emission levels than the selfish ones. It may arise that $\mu=1$ for low types.

Using (2), we can substitute the right hand side of (8) for $\mu$ in program II to obtain

$$
\begin{aligned}
& \max _{q(\cdot), R(\cdot), K} \int_{\underline{\theta}}^{\bar{\theta}}\{(1+\lambda)(\pi(q(\theta), \theta)-c(K+R(\theta)-\pi(q(\theta), \theta)) / \bar{F})-d q(\theta)-\lambda R(\theta)\} g(\theta) d \theta \\
& \text { s.t. } \\
& \dot{R}(\theta)=\partial_{\theta} \pi(q(\theta), \theta) \\
& R(\underline{\bar{\theta}})=0 \\
& R(\bar{\theta})-\pi^{\circ}(\bar{\theta})+K \geq 0
\end{aligned}
$$

where constraint $\mu \leq 1$ is neglected, i.e.

$$
R(\theta)-\pi(q(\theta), \theta)+K \leq \bar{F}
$$

Program III is a parameterized optimal control problem (with $K$ as the parameter), where informational rent $R(\cdot)$ stands for the state variable and emission schedule $q(\cdot)$ for the control variable. Since $K$ affects the objective of program III negatively, the 
last inequality of this program is binding at the optimum. The resolution of program III is given in the appendix. This solution solves agency problem I if the monotonicity constraint on $q(\cdot)$ and conditions $0 \leq q(\cdot)<q^{\circ}(\cdot)$ and (10) are satisfied. We shall go back to these constraints after having characterized the unconstrained emission schedule given in the following proposition.

Proposition 3 The solution of program III, emission schedule $\hat{q}(\cdot)$, satisfies

$$
\partial_{q} \pi(\hat{q}(\theta), \theta)=\frac{d}{1+\lambda}-\frac{c}{\bar{F}} \partial_{q} \pi(\hat{q}(\theta), \theta)+M(\theta) \partial_{\theta q} \pi(\hat{q}(\theta), \theta)
$$

where

$$
M(\theta) \equiv \frac{\lambda}{1+\lambda} \frac{1-G(\theta)}{g(\theta)}-\frac{c}{\bar{F}} \frac{G(\theta)}{g(\theta)}
$$

Proof. See appendix.

Compared to perfect information levels, the emission schedule involves two additional terms in the right hand side of (11), one reflects the evasion problem and the other is due to the adverse selection problem. To interpret (11), it is useful to consider the two benchmark cases of a costly audit-cum-perfect information and a free audit-cum-asymmetric information. Depicted in Fig. 1 are the emission schedules corresponding to these different assumptions.

To understand the direct effect of the evasion problem on the emissions schedule, assume first that the regulator has perfect knowledge of firms' types but that the agency has to audit each firm to discover their emission levels. Denote by $e(\cdot)$ the optimal emission schedule in that case. Since deterring imitation is not relevant, (IC1) constraints do not matter. We have $R(\theta)=0$, i.e. $t(\theta)=\pi(e(\theta), \theta)$. However, the agency still has to deter evasion, i.e.; that firms pay the tax amounts corresponding to their type and pollute accordingly. When eliminating the term due to adverse selection in (11), the optimal emission schedule satisfies

$$
\partial_{q} \pi(e(\theta), \theta)=\frac{d}{1+\lambda} \frac{1}{1+c / \bar{F}}
$$


and thus $e(\cdot)>q^{*}(\theta)$, i.e.; we have under-deterrence compared to first-best levels. Indeed, to deter any deviation of firms from their assigned emission levels, the agency has to inspect the type- $\theta$ firm at a minimal rate $\mu(\theta)=(K-\pi(q(\theta), \theta)) / \bar{F}$. Compared to the perfect information level, a $d q$ increase in the type- $\theta$ emission level induces a decrease in the inspection cost of $(d / d q)[c \mu]=-\partial_{q} \pi\left(q^{*}(\theta), \theta\right) c / \bar{F}$. The corresponding marginal social loss is $(1+\lambda) \partial_{q} \pi\left(q^{*}(\theta), \theta\right)-d=0$. The agency will thus increase the emission schedule above that of perfect information.

Second, assume that audit is free $(c / \bar{F}$ is negligible) but does not reveal the firms' types. We would have the usual adverse-selection quantity schedule (à la Baron and Myerson, 1982, B-M hereafter), given by

$$
\partial_{q} \pi\left(q^{B M}(\theta), \theta\right)=\frac{d}{1+\lambda}+\frac{\lambda}{1+\lambda} \frac{1-G(\theta)}{g(\theta)} \partial_{\theta q} \pi\left(q^{B M}(\theta), \theta\right)
$$

which states that, due to asymmetric information, the optimal emission schedule is lower than the perfect information one except for firms with the highest type, as depicted in Fig. 1. In that case, compared to the perfect information level, the principal has indeed to trade-off the social welfare gain of a $d q$ increase in emissions for $g(\theta)$ type- $\theta$ firms, equal to $(1+\lambda) \partial_{q} \pi\left(q^{*}(\theta), \theta\right)-d=0$, with the social losses of decreased tax collections (increased informational rents) on all firms of type higher than $\theta$, that amount to $(1-G(\theta)) \lambda \partial_{\theta q} \pi\left(q^{*}(\theta), \theta\right)>0$. The optimal schedule solution of this tradeoff $q^{B M}(\cdot)$, allows the agency to deter firms of type higher than a given $\theta$ to mimic the type- $\theta$ firm. Observe that this adverse-selection effect induces marginal tax rates that decrease with the firms' type and that the most efficient firms only have a marginal tax rate equal to the perfect information level.

In the general case where the audit is costly and reveals only the firms' emission levels, there is an additional distortion that affects the emission schedule. It is equal to

$$
-(1+\lambda) c / \bar{F} G(\theta) \partial_{\theta q} \pi(q(\theta), \theta)
$$


and can be interpreted as follows. First, in addition to the usual mimicking adverseselection effects produced by a $d q$ increase in the emission level of the type- $\theta$ firm, the increase indirectly changes the inspection efforts on higher types. Indeed, to maintain the same expected penalty when reporting a type greater than $\theta$, the agency has to increase the inspection efforts on these firms: since the $d q$ increase induces a $d R$ increase for all firms with type greater than $\theta$ and have $d \mu / d R=1 / \bar{F}$ for all $\theta$ (recall that increased rents correspond to lower tax burdens). This induces an additional marginal social cost equal to

$$
(1+\lambda) c / \bar{F}(1-G(\theta)) \partial_{\theta q} \pi(q(\theta), \theta)
$$

However, the increase in the type- $\bar{\theta}$ informational rent allows the agency to reduce the expected cost of evading $K$, since we have $R(\bar{\theta})=\pi^{\circ}(\bar{\theta})-K$ at the optimum, i.e. at the optimum, evading or paying their taxes must be indifferent to these firms. In turn, this decrease induces a lower inspection rate for all firms (since $d \mu / d K=1 / \bar{F}$ ). The marginal saving of inspection costs is given by

$$
(1+\lambda) c / \bar{F} \partial_{\theta q} \pi(q(\theta), \theta)
$$

Consequently, inspection efforts are unaffected for the firms with type higher than $\theta$ (the increase in their rents $R(\cdot)$ and the decrease in $K$ let $\mu(\cdot)$ unchanged), but they are reduced for types lower than $\theta$ (the decrease in $K$ is not compensated by an increase in their informational rents). To sum it up, with costly enforcement and self-reporting, the agency has to account for two opposite adverse-selection effects: under-deterrence increases the informational rents of the most profitable firms, but greater informational rents allow for a decrease in the inspection effort on less efficient firms. The result of the agency's trade-off is an emission schedule that is steeper than the previous ones, as depicted in Fig. 1. Observe that mimicking and shirking adverseselection effects distort the emission schedule in opposite directions around emission 
levels $e(\theta)$, which the regulator would implement if he knew the firms' type when taking the audit costs into account. As depicted in Fig. 1, there is a unique firm's type $\theta_{s}$ for which these adverse-selection effects annihilate each other, i.e.; $M\left(\theta_{s}\right)=0$ which implies $\hat{q}\left(\theta_{s}\right)=e\left(\theta_{s}\right)$. More generally, we have the following results:

Corollary 4 Emission schedule $\hat{q}(\cdot)$ solution of program III satisfies

(i) For all $\theta<\theta_{s}, \hat{q}(\theta)<e(\theta)$ and for all $\theta>\theta_{s}, \hat{q}(\theta)>e(\theta)$, where $\theta_{s}$ is given by

$$
\theta_{s}=G^{-1}\left(\frac{\lambda}{\lambda+(1+\lambda) c / \bar{F}}\right)
$$

and we have $\underline{\theta}<\theta_{s}<\bar{\theta}$, $d \theta_{s} / d \lambda>0$ and $d \theta_{s} / d[c / \bar{F}]<0$.

(ii) $\hat{q}(\underline{\theta})<q^{*}(\underline{\theta})$ if $\partial_{\theta q} \pi\left(q^{*}(\underline{\theta}), \underline{\theta}\right)>c / \bar{F} g(\underline{\theta}) / \lambda$.

(iii) $\hat{q}(\bar{\theta})=q^{\circ}(\bar{\theta})$ if $\partial_{\theta q} \pi\left(q^{\circ}(\bar{\theta}), \bar{\theta}\right) \geq g(\underline{\theta}) /((1+\lambda) c / \bar{F})$.

Proof. See appendix.

By (i), the mimicking effect dominates evasion for low profitability firms. The agency thus designs a tax schedule that induces these firms to under-pollute compared to schedule $e(\cdot)$. If the marginal profit of pollution increases rapidly with the type at perfect information emission levels $q^{*}(\cdot)$, this mimicking effect may lead the agency to enforce pollution levels below perfect information emission levels for low types as stated in point (ii). This is the case in Fig. 1 for all types lower than $\theta_{2}$, where all firms bellow $\theta_{1}$ have their emissions reduced to zero. For more profitable firms, the shirking effect is dominant and leads the agency to induce higher emission levels than $e(\cdot)$. As revealed in point (iii), when the marginal profit of pollution increases rapidly, the agency may even give up pollution reduction for the higher types. ${ }^{16}$ This case is also depicted in Fig. 1 where all firms with the emission levels of all firms with types greater than $\theta_{3}$ have their equal their private optimum $q^{\circ}(\theta)$.

\footnotetext{
${ }^{16}$ It may even be socially optimal to induce the more profitable firms to over-pollute compared to their selfish levels, see footnote 14 .
} 
It is interesting at this point to comment briefly on the pattern of the optimal policy in the case of negligible second-dividend aspects. When $\lambda=0$, informational rents $R(\theta)$ are no longer directly socially costly. However, they still affect monitoring efforts and thus are indirectly costly, through pollution monitoring cost, as the objective of program III indicates. Indeed, greater informational rents correspond to lower tax payments. When tax payments are low, the agency has to increase the probability of inspection to maintain the same expected payment for a would-be evading firm. Consequently, inspection probabilities and informational rents are positively related and the regulator still has an incentive to reduce rents in order to decrease monitoring cost. As a result, when $\lambda=0$, the mimicking adverse-selection effect disappears but the shirking adverse-selection effect still matters in the determination of the optimal pollution level. This appears clearly in equation (11), which can be written as

$$
\partial_{q} \pi(\hat{q}(\theta), \theta)=d-\frac{c}{\bar{F}} \partial_{q} \pi(\hat{q}(\theta), \theta)-\frac{c}{\bar{F}} \frac{G(\theta)}{g(\theta)} \partial_{\theta q} \pi(\hat{q}(\theta), \theta)
$$

Observe that the first-best taxation rule $\left(\partial_{q} \pi=d\right)$ must still be corrected by two terms. Both the impact of deterring tax evasion and the shirking adverse-selection effect lead the regulator to implement higher emission levels than the first-best ones.

Finally, in the general case, when (10) does not bind, that is, when inspection rate $\mu$ is inferior to 1 everywhere, emission schedule $e^{*}(\cdot)$ solution of program I is given by $e^{*}(\theta)=\hat{q}(\theta)$ for all $\theta \in \Theta$ (whenever $0 \leq \hat{q}(\theta) \leq q^{\circ}(\theta)$ ). To complete the analysis, the last proposition characterizes the opposite case of an inspection rate $\mu$ equal to 1 for the low types firms. As stated formally, the entire regulation schedule is affected, with every firm's emission levels larger than in the unconstrained case.

Proposition 5 If (10) binds on a $\Theta$ subset, the solution of program I, emission schedule $e^{*}(\cdot)$, verifies $e^{*}(\theta)>\hat{q}(\theta)$ for all $\theta \in \Theta$ (whenever $\hat{q}(\theta)<q^{\circ}(\theta)$ ).

Proof. See appendix. 
It remains to verify that the second-order conditions for an incentive-compatible policy are satisfied. Differentiating (11) gives

$$
\begin{aligned}
0= & {\left[(1+c / \bar{F}) \partial_{q q} \pi(\hat{q}(\theta), \theta)-M(\theta) \partial_{\theta q q} \pi(\hat{q}(\theta), \theta)\right] \hat{q}^{\prime}(\theta) } \\
& -M(\theta) \partial_{\theta \theta q} \pi(\hat{q}(\theta), \theta)-\left[M^{\prime}(\theta)-(1+c / \bar{F})\right] \partial_{\theta q} \pi(\hat{q}(\theta), \theta)
\end{aligned}
$$

With inverse hazard rates, i.e. $d / d \theta[G(\theta) / g(\theta)]>0$ and $d / d \theta[(1-G(\theta)) / g(\theta)], M(\cdot)$ is non-increasing. However, since $M(\underline{\theta})>0$ and $M(\bar{\theta})<0$, the sign of $\hat{q}^{\prime}(\theta)$ is ambiguous, even under the usual assumptions about third derivatives of agent's profit function which are commonly made in standard adverse selection problems. Consequently, the second-order conditions on the emission schedule are more likely to be binding when the emissions are costly to observe than when assuming perfect observation of pollution levels. ${ }^{17}$

However, in the particular case of profit functions linear in $\theta$, e.g. $\pi(q, \theta)=\theta B(q)$ where $B$ is an increasing and a concave function of $q$ satisfying $B^{\prime}(0)=+\infty$, monotonic hazard rates are sufficient to insure an increasing emission schedule. Indeed, in that case we have $\hat{q}(\theta)>0$ for all $\theta$ only if

$$
1+\frac{c}{\bar{F}}>M(\underline{\theta}) / \underline{\theta}
$$

or equivalently

$$
\underline{\theta}(\underline{\theta})>\frac{\lambda}{1+\lambda} \frac{1}{1+c / \bar{F}}
$$

that also implies $\hat{q}^{\prime}(\theta)>0$ using (13). If (14) is not satisfied, firms with type $\theta<\theta_{1}$ will choose not to pollute (i.e.; we have $\hat{q}(\theta)=0$ for all $\theta \leq \theta_{1}$ ), with $\theta_{1}$ satisfying

$$
(1+c / \bar{F}) \theta_{1}-M\left(\theta_{1}\right)=0
$$

and the second-order condition is trivially satisfied for all $\theta<\theta_{1}$.

\footnotetext{
${ }^{17}$ When the monotonicity constraints on the emission schedule bind, the optimal solution entails the bunching of individuals (see Guesnerie and Laffont, 1984, for a formal treatment).
} 
To complete the analysis, it is worth considering the case of pollution's marginal benefits which decrease with firms' profitability parameter $\theta$, i.e.; $\pi_{q \theta}<0$ (together with $\left.\pi_{\theta}>0\right)$. Compared to the previous analysis, the optimal emission schedule decreases according to type. Moreover, in that case, the least profitable firms are the most tempted by tax evasion. Indeed, it is easy to show that the constraint corresponding to (IC2') is $R(\underline{\theta})-\pi^{\circ}(\underline{\theta})+K \geq 0$. As (IR') is still binding at the optimum, this evasion constraint simplifies to $K \geq \pi^{\circ}(\underline{\theta})$ and is binding at the optimum since $K$ affects the regulator's objective negatively. On the other hand, the other firms are still able to extract informational rents by understating their types. This implies that the adverse selection term in (11) has the opposite sign, and thus the adverse selection effects described above distort the optimal emission schedule in opposite directions.

\section{Environmental standard policy}

In this section, we investigate the properties of the typical command-and-control policy of a pollution standard enlarged to its enforcement aspects. As we will show, an increasing fine schedule allows the agency to introduce efficiency considerations in an otherwise rather inefficient policy. The standard policy then appears to be a restricted case of a taxation policy because of the "marginal deterrence" induced by the penalty schedule of its enforcement policy. To make this point more striking, we consider the simplest case of a standard policy where the firms do not report their emission levels to the agency: They simply pay a licence fee $k$ that allows them to pollute up to an uniform quota $z$. Since the firms' pollution $q$ levels are not reported to the agency and because the agency cannot distinguish between firms, it audits all firms with the same inspection rate $\mu$. The task of the agency is thus to decide on a $z$ standard level, a $k$ licence and a fine schedule $f(q)$ (along with a monitoring rate $\mu$ ), where $f(q) \geq 0$ whenever the audit reveals a $q$ emission level greater than $z$. In the following, standard $z$ will be implicitly defined as the maximal emission level with a null fine. 
Thanks to the taxation principle, we can use the mechanism-design approach and consider that a uniform standard policy is given by $\{z, k, \mu, F(\tilde{\theta}), q(\tilde{\theta}): \tilde{\theta} \in \Theta\}$ where $\tilde{\theta}$ is the firm's announcement, $q(\tilde{\theta})$ and $F(\tilde{\theta})=f(q(\tilde{\theta}))$ two functions of this announcement, and $z, k, \mu$ are three constants. The interpretation of this mechanism reads as follow: The firm truthfully reports its type $\theta$, and pollutes $q(\theta)$ which may be greater or lower than standard $z$. Whenever $q(\theta)$ is lower than $z$, it pays only a lump sum licence $k$, whereas it is charged an expected amount $k+\mu F(\theta)$ for levels greater than $z$. The monitoring rate is constrained to be the same for all firms, and we have $F(\tilde{\theta})=0$ whenever $q(\tilde{\theta}) \leq z$.

Inflicting the maximum fine whenever the firms exceed the standard is no longer socially efficient. Indeed, fines proportional to the fraud may induce profitable firms to pollute over the standard and thus restore the efficiency of the environmental policy. In that case, the expected fine $\mu F(\tilde{\theta})$ for pollution $q(\tilde{\theta})>z$ is close to an environmental tax, as discussed in the previous section. ${ }^{18}$

The type- $\theta$ firm's expected profit with a standard policy is given by

$$
u(\theta, \tilde{\theta})=\pi(q(\tilde{\theta}), \theta)-k-\mu F(\tilde{\theta})
$$

and the schedule is incentive-compatible if, for all $\theta \in \Theta$,

$$
R(\theta) \equiv u(\theta, \theta) \geq u(\theta, \tilde{\theta})
$$

and

$$
R(\theta) \geq \max _{q} \pi(q, \theta)-k-\mu \bar{F}
$$

We also have to take account of (IR) constraints. However, it may be worthwhile when defining the standard policy to exclude (implicitly) the lower profitability firms

\footnotetext{
${ }^{18} \mathrm{~A}$ standard corresponds to the particular (and constrained) case of a taxation policy. The mechanism proposed is truly stochastic, since payment depends on the probability of inspection $\mu$. In the unconstrained taxation case, the payment structure is $p(\theta)=t(\theta)+\mu(\theta) f(\theta, q(\theta))=t(\theta)$, whereas it is given by $p(\theta)=k+\mu F(\theta)$ (for all $\theta$ where $q(\theta)>0$ ) in the case of a standard. The mechanism is thus constrained by $t(\theta)=k$ and $\mu(\theta)=\mu$, and economic incentives are given through the expected penalty $\mu F(\theta)$ only.
} 
by charging a large fee. Indeed, there is an obvious trade-off between the licence fee charged and the number of firms that can afford to pay it. As a consequence, for a given $k$ licence, only firms with type greater than threshold level $\theta_{0} \geq \underline{\theta}$ benefit from polluting, and we have $R\left(\theta_{0}\right)=0$.

Taking the licence and the fine schedule into account, the social welfare becomes

$$
W=\int_{\theta_{0}}^{\bar{\theta}}\{\pi(q(\theta), \theta)-d q(\theta)+\lambda(k+\mu F(\theta))\} g(\theta) d \theta-(1+\lambda) c \mu
$$

Using previous arguments (see lemma 1), an incentive mechanism must satisfy

$$
\dot{R}(\theta)=\partial_{\theta} \pi(q(\theta), \theta)
$$

along with non-decreasing emission schedule $q(\cdot)$ and

$$
R(\bar{\theta}) \geq \pi^{\circ}(\bar{\theta})-k-\mu \bar{F}
$$

which is binding at the optimum of the agency's program and defines $\mu$. The fine is deduced from (15) according to the relation

$$
\mu F(\theta)=\pi(q(\theta), \theta)-R(\theta)-k
$$

and since $0 \leq F(\cdot) \leq \bar{F}$, we must have

$$
0 \leq \pi(q(\theta), \theta)-R(\theta)-k \leq \mu \bar{F}
$$

for all $\theta$, where the last inequality is satisfied whenever (16) holds (and thus whenever (18) holds).

The agency's program is thus given by

$$
\begin{aligned}
& \max _{q(\cdot), R(\cdot), k, \theta_{0}} \int_{\theta_{0}}^{\bar{\theta}}\{(1+\lambda) \pi(q(\theta), \theta)-d q(\theta)-\lambda R(\theta)\} g(\theta) d \theta-(1+\lambda) \frac{c}{\bar{F}}\left(\pi^{\circ}(\bar{\theta})-R(\bar{\theta})-k\right) \\
& \text { s.t. } \\
& \dot{R}(\theta)=\partial_{\theta} \pi(q(\theta), \theta) \\
& R\left(\theta_{0}\right)=0 \\
& \pi(q(\theta), \theta)-R(\theta)-k \geq 0 \\
& \pi^{\circ}(\bar{\theta})-R(\bar{\theta})-k \leq \bar{F}
\end{aligned}
$$


where $\theta_{0} \geq \underline{\theta}$ and where the monotonicity condition on the emission schedule $q(\cdot)$ is neglected and must thus be verified afterwards.

Program IV is a simple parametrized optimal control problem, where $R(\cdot)$ stands for the state variable, $q(\cdot)$ for the control variable and $k$ for the parameter. The solution of this program is characterized in the following proposition, assuming that the last constraint is slack (i.e. $\mu<1$ ).

Proposition 6 Assuming an interior solution in $\mu$, the solution of program IV satisfies

(i) $k=\pi\left(z, \theta_{0}\right)>0$.

(ii) $F(\theta)=0$ and $q(\theta)=z<q^{\circ}\left(\theta_{0}\right)$ for all $\theta_{0} \leq \theta \leq \hat{\theta}$ with $\hat{\theta}>\theta_{0}$.

(iii) $z$ and $\hat{\theta}$ satisfy

$$
\begin{aligned}
0= & -\lambda\left(1-G\left(\theta_{0}\right)\right) \frac{\partial_{q} \pi\left(z, \theta_{0}\right)}{\partial_{q} \pi(z, \hat{\theta})}+d \frac{G(\hat{\theta})-G\left(\theta_{0}\right)}{\partial_{q} \pi(z, \hat{\theta})}-\int_{\theta_{0}}^{\hat{\theta}} \frac{\partial_{q} \pi(z, x)}{\partial_{q} \pi(z, \hat{\theta})} g(x) d x \\
& -(1+\lambda) \frac{c}{\bar{F}}+\lambda(1-G(\hat{\theta})) \\
0= & \left((1+\lambda) \partial_{q} \pi(z, \hat{\theta})-d\right) g(\hat{\theta})+\left((1+\lambda) \frac{c}{\bar{F}}-\lambda(1-G(\hat{\theta}))\right) \partial_{\theta q} \pi(z, \hat{\theta})
\end{aligned}
$$

with $\theta_{0} \geq \underline{\theta}$, and if $\theta_{0}>\underline{\theta}$ we have

$$
0=(1+\lambda) \pi\left(z, \theta_{0}\right)-d z
$$

(iv) For all $\theta>\hat{\theta}, q(\theta)$ satisfies

$$
\begin{aligned}
\partial_{q} \pi(q(\theta), \theta)= & \frac{d}{1+\lambda}-\frac{c}{\bar{F}} \frac{1}{g(\theta)} \partial_{\theta q} \pi(q(\theta), \theta) \\
& +\frac{\lambda}{1+\lambda} \frac{1-G(\theta)}{g(\theta)} \partial_{\theta q} \pi(q(\theta), \theta)
\end{aligned}
$$

Proof. See appendix.

By (i), it is socially efficient to impose a positive license fee on firms. The fee corresponds to the benefit the least profitable firms obtain from polluting at the standard. These firms thus do not benefit from the policy, while firms of lower type, if any, 
choose not to pollute because the benefit from polluting up to the standard is smaller than the fee they have to pay. As revealed by (ii), up to an intermediate profitability type $\hat{\theta}$, low profitable firms pollute at the standard in accordance to their licence and consequently are not fined when inspected. For these firms the standard corresponds to a real constraint compared to the levels they would have chosen without regulation. More profitable firms exceed the standard. The emission levels they choose is given by (20). These levels result from the agency trade-offs between adverse selection costs and audit costs. Indeed, as observed previously, if $\lambda=0$, the inspection cost induces an increase in the emission levels compared to those of perfect information. On the other hand, if $c / \bar{F}$ is negligible, the adverse selection effect is dominant, and the emission levels are lower than with perfect information for all but the most profitable firms. Consequently, depending on the efficiency of the firm, the marginal expected penalty for violating the standard can be higher or lower than the marginal damage.

Again, one can easily verify by differentiating (20) that the usual assumptions about third derivatives of the profit function and on hazard rates are not sufficient to guarantee an increasing pollution level. The optimal policy may thus involve a bunching of individuals on subset $[\hat{\theta}, \bar{\theta}]$.

\section{Conclusion}

We have studied the optimal environmental taxation and standard policies under asymmetric information with an imperfect and costly audit. Compared to the results of Baron (1985) and Laffont (1994), we have shown that the threat of being audited alter the usual incentives of firms to over-estimate their abatement costs. In particular, depending on firms' abatement costs, the optimal policy may involve over or underdeterrence compared to perfect information levels. We also showed that a pollution standard is close to an environmental tax once the economic incentives of the accompanying enforcement policy are considered. 
The main policy implications of our analysis are the following. Environmental quality and fiscal considerations are conflicting objectives. The textbook Pigovian tax allows the regulator to raise some revenue while reducing pollution to efficient levels. Assuming that firms do not evade, the agency does not have to worry about the firms' private information to obtain some tax revenue from the policy. However, the environmental efficiency of the policy is severely reduced if the regulator primarily wants to raise tax revenue with an environmental tax. The environmental quality is reduced since the agency grants more allowances to pollute in order to increase the tax base. Moreover, the environmental policy suffers from the usual woes of the other tax systems: Tax evasion and inefficient uses of plants or production facilities reduce the tax burden. The regulator has thus to design an incentive tax schedule to enhance economic efficiency and to perform costly monitoring activities to enforce the policy. We demonstrated that deterring evasion through pollution monitoring and screening heterogenous firms through taxation are intimately related. From a fiscal standpoint, environmental taxes are more efficient than standards. Informational rents left to firms are larger under an environmental standard than with a taxation policy. Whether this result extends to the case of tradable pollution permits remains an open question and needs further research. However, from an environmental standpoint, standards may appear more efficient. Indeed, as shown in an illustrative example in Bontems and Bourgeon (2002), total pollution may be lower under the optimal standard than under the optimal tax. These computations also show that administrative costs may be greater with a taxation policy than with a standard.

Finally, as usual in most models of audit with commitment, the tax policy analyzed here suffers from a time inconsistency problem. Indeed, no audit is needed ex-post as all firms are compliant with the optimal pollution scheme. In the case where the agency's inspection effort is not readily verifiable by firms, such a commitment seems unrealistic (for more on this problem, see Khalil, 1997). This is not the case for the 
command-and-control policy of a pollution standard. Indeed, the monitoring of the firms allows the regulator to raise some revenues in addition to the licence fees in case of standard violations. 


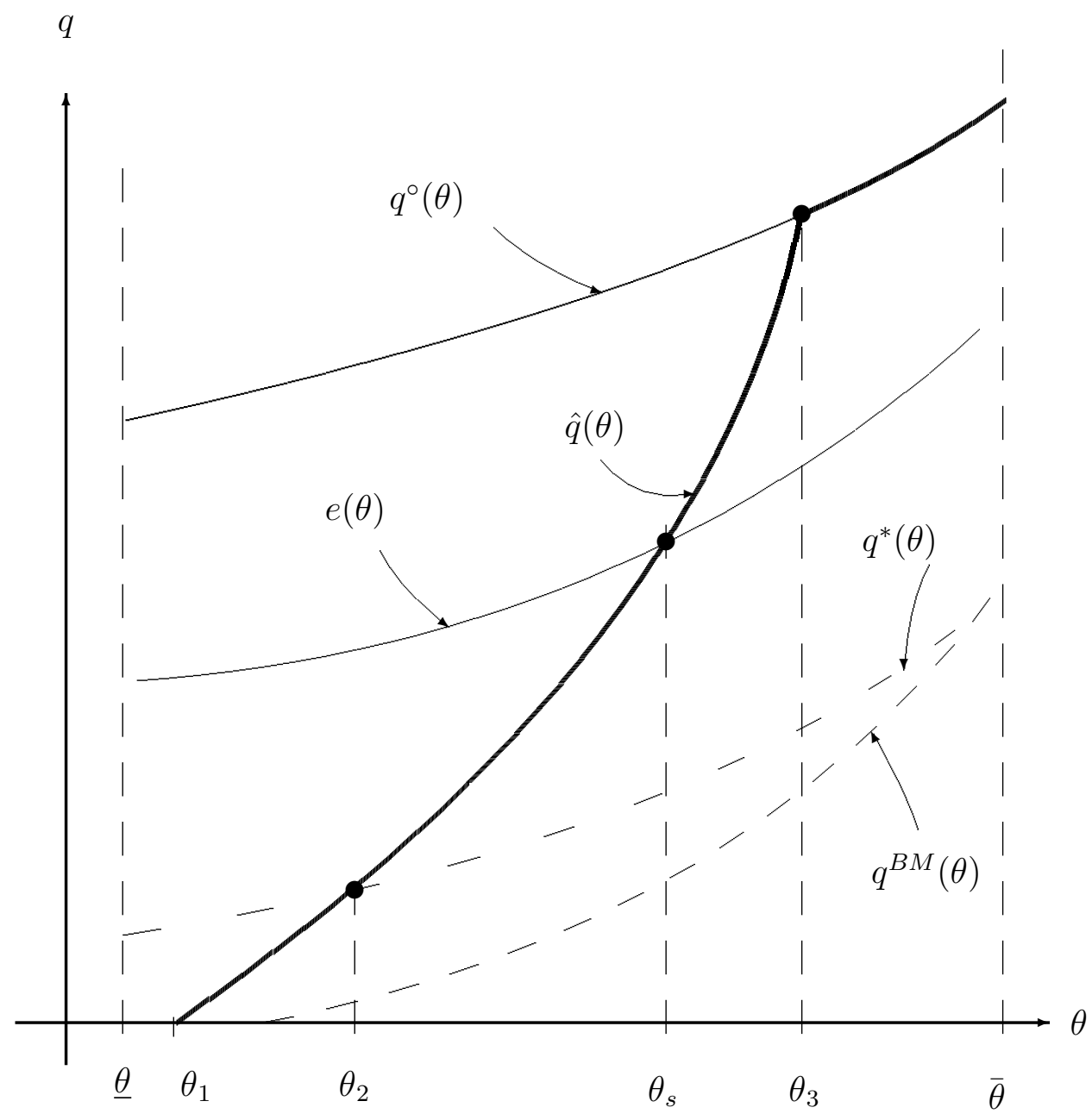

Figure 1: Optimal emission schedule and asymmetric information. 


\section{References}

[1] Baron D., 1985, Regulation of Prices and Pollution under Incomplete Information, Journal of Public Economics, 28, 211-231.

[2] Baron D. and D. Besanko, 1984, Regulation, Asymmetric Information and Auditing, Rand Journal of Economics, 15 (4), 447-470.

[3] Baron D. and R. Myerson, 1982, Regulating a Monopolist with Unknown Costs, Econometrica, 50, 911-930.

[4] Bontems P. and J.M. Bourgeon, 2002, Optimal Environmental Taxation and Enforcement Policy, THEMA working paper.

[5] Border K.C. and J. Sobel, 1987, Samurai Accountant: A Theory of Auditing and Plunder, Review of Economic Studies, 54, 525-540.

[6] Boyer M. and J.J. Laffont, 1997, Environmental Risks and Bank Liability, European Economic Review, 41( 8), 1427-59.

[7] Chander P. and L.L. Wilde, 1998, A General Characterization of Optimal Income Tax Enforcement, Review of Economic Studies, 65, 165-183.

[8] Cohen M.A. (1999), Monitoring and Enforcement of Environmental Policy, in: Folmer H. and Tietenberg T., eds, The International Yearbook of Environmental Resource Economics 1999/2000. Cheltenham, Edward Elgar Publishing.

[9] Cremer H. and F. Gahvari, 1996, Tax Evasion and the Optimal General Income Tax, Journal of Public Economics, 60, 235-249.

[10] European Environmental Agency, 1996, Environmental Taxes : Implementation and Environmental Effectiveness, Environmental Issues Series, n ${ }^{\circ}$, Copenhagen. 
[11] Guesnerie R. and J.J. Laffont, 1984, A Complete Solution to a Class of PrincipalAgent Problems with an Application to the Control of a Self-Managed Firm, Journal of Public Economics, 25, 329-369.

[12] Jullien B., 2000, Participation Constraints in Adverse Selection Models, Journal of Economic Theory, 93, 1-47.

[13] Khalil F., 1997, Auditing without commitment, Rand Journal of Economics, 28, 629-640.

[14] Laffont J.J., 1994, Regulation of Pollution with Asymmetric Information, chapter 2 in Nonpoint Source Pollution Regulation: Issues and Analysis, C. Dosi and T. Tomasi, eds., Kluwer Academic Publishers, Dordrecht.

[15] Laffont J.J.and J. Tirole, 1993, A Theory of Incentives in Procurement and Regulation, (MIT Press, Cambridge).

[16] Lewis, T. R., 1996, Protecting the Environment When Costs and Benefits are Privately Known, Rand Journal of Economics, 27, 819-847.

[17] Malik A.S., 1992, Enforcement Costs and the Choice of Policy Instruments for Controlling Pollution, Economic Inquiry, 30, 714-721.

[18] Mookherjee D. and I.P.L. Png, 1994, Marginal Deterrence in Enforcement of Law, Journal of Political Economy, 102, 1039-1066.

[19] Swierzbinski J.E., 1994, Guilty Until Proven Innocent - Regulation with Costly and Limited Enforcement, Journal of Environmental Economics and Management, 27, 127-146. 


\section{Appendix}

\section{A Proof of proposition 3}

With $\nu(\cdot)$ as the Lagrange multipliers for (IC1') and by $\tau \geq 0$ the multiplier for (IC2'), the Lagrangian of program III is given by

$$
\begin{aligned}
\mathcal{L}= & \int_{\underline{\theta}}^{\bar{\theta}}\left\{(1+\lambda)\left(1+\frac{c}{\bar{F}}\right) \pi(q(\theta), \theta)-d q(\theta)-\left(\lambda+(1+\lambda) \frac{c}{\bar{F}}\right) R(\theta)\right\} g(\theta) d \theta \\
& -(1+\lambda) K \frac{c}{\bar{F}}+\int_{\underline{\theta}}^{\bar{\theta}} \nu(\theta)\left(\partial_{\theta} \pi(q(\theta), \theta)-\dot{R}(\theta)\right) d \theta+\tau\left(R(\bar{\theta})-\pi^{\circ}(\bar{\theta})+K\right)
\end{aligned}
$$

Integrating by parts gives

$$
\int_{\underline{\theta}}^{\bar{\theta}} \nu(\theta) \dot{R}(\theta) d \theta=\nu(\bar{\theta}) R(\bar{\theta})-\nu(\underline{\theta}) R(\underline{\theta})-\int_{\underline{\theta}}^{\bar{\theta}} \dot{\nu}(\theta) R(\theta) d \theta
$$

and the Lagrangian reduces itself to

$$
\mathcal{L}=\int_{\underline{\theta}}^{\bar{\theta}} H(\theta) d \theta-\left((1+\lambda) \frac{c}{\bar{F}}-\tau\right) K-R(\bar{\theta})(\nu(\bar{\theta})-\tau)-\tau \pi^{\circ}(\bar{\theta})
$$

where

$$
\begin{aligned}
H \equiv & ((1+\lambda)(1+c / \bar{F}) \pi(q(\theta), \theta)-d q(\theta)) g(\theta) \\
& +R(\theta)\{\dot{\nu}(\theta)-(\lambda+(1+\lambda) c / \bar{F}) g(\theta)\}+\nu(\theta) \partial_{\theta} \pi(q(\theta), \theta)
\end{aligned}
$$

Assuming an interior solution, pointwise maximizations give

$$
\begin{aligned}
\frac{\partial H}{\partial q}= & 0=g(\theta)\left((1+\lambda)(1+c / \bar{F}) \partial_{q} \pi(q(\theta), \theta)-d\right) \\
& +\nu(\theta) \partial_{\theta q} \pi(q(\theta), \theta) \\
\frac{\partial H}{\partial R}= & \dot{\nu}(\theta)-(\lambda+(1+\lambda) c / \bar{F}) g(\theta)=0
\end{aligned}
$$

with transversality condition

$$
\frac{\partial \mathcal{L}}{\partial R(\bar{\theta})}=\tau-\nu(\bar{\theta})=0
$$


and with first-order condition for $K$ given by

$$
\frac{\partial \mathcal{L}}{\partial K}=\tau-(1+\lambda) \frac{c}{\bar{F}}=0
$$

Integrating (22) gives

$$
\nu(\bar{\theta})-\nu(\theta)=(\lambda+(1+\lambda) c / \bar{F})(1-G(\theta))
$$

where

$$
\nu(\bar{\theta})=(1+\lambda) c / \bar{F}
$$

using (23) and (24). We thus have

$$
\begin{aligned}
\nu(\theta) & =(1+\lambda) c / \bar{F}-(\lambda+(1+\lambda) c / \bar{F})(1-G(\theta)) \\
& =(1+\lambda) c / \bar{F} G(\theta)-\lambda(1-G(\theta))
\end{aligned}
$$

Plugging this expression into (21) and rearranging terms gives (11).

\section{B Proof of corollary 4}

Point (i) is straightforward. We have $\hat{q}(\underline{\theta})<q^{*}(\underline{\theta})$ if $\left.(\partial H / \partial q)\right|_{\theta=\underline{\theta}, q=q^{*}}<0$ and $\hat{q}(\bar{\theta})=$ $q^{\circ}(\bar{\theta})$ if $\left.(\partial H / \partial q)\right|_{\theta=\bar{\theta}, q=q^{\circ}} \geq 0$. Using (25) and (21),

$$
\left.\frac{\partial H}{\partial q}\right|_{\theta=\underline{\theta}, q=q^{*}}=g(\underline{\theta})\left((1+\lambda)(1+c / \bar{F}) \partial_{q} \pi\left(q^{*}(\underline{\theta}), \underline{\theta}\right)-d\right)-\lambda \partial_{\theta q} \pi\left(q^{*}(\underline{\theta}), \theta\right)
$$

and

$$
\left.\frac{\partial H}{\partial q}\right|_{\theta=\bar{\theta}, q=q^{\circ}}=g(\bar{\theta})\left((1+\lambda)(1+c / \bar{F}) \partial_{q} \pi\left(q^{\circ}(\bar{\theta}), \bar{\theta}\right)-d\right)+(1+\lambda) c / \bar{F} \partial_{\theta q} \pi\left(q^{\circ}(\bar{\theta}), \theta\right)
$$

where $\partial_{q} \pi\left(q^{*}(\underline{\theta}), \underline{\theta}\right)=d /(1+\lambda)$ and $\partial_{q} \pi\left(q^{\circ}(\bar{\theta}), \bar{\theta}\right)=0$ by definition, which gives (ii) and (iii). 


\section{Proof of proposition 5}

With $\gamma(\theta) \geq 0$ as the Lagrange multiplier for constraints (10), first-order conditions (21)-(24) become

$$
\begin{aligned}
\frac{\partial H}{\partial q}= & 0=g(\theta)\left((1+\lambda)(1+c / \bar{F}) \partial_{q} \pi(q(\theta), \theta)-d\right) \\
& +\rho(\theta) \partial_{\theta q} \pi(q(\theta), \theta)+\gamma(\theta) \partial_{q} \pi(q(\theta), \theta) \geq 0\left(q \leq q^{\circ}\right) \\
\frac{\partial H}{\partial R}= & \dot{\rho}(\theta)-(\lambda+(1+\lambda) c / \bar{F}) g(\theta)-\gamma(\theta)=0 \\
\frac{\partial \mathcal{L}}{\partial R(\bar{\theta})}= & \tau-\rho(\bar{\theta})=0 \\
\frac{\partial \mathcal{L}}{\partial K}= & \tau-(1+\lambda) \frac{c}{\bar{F}}-\int_{\Theta} \gamma(\theta) d \theta=0 .
\end{aligned}
$$

where $\rho(\cdot)$ is the costate variable. Integrating $(27)$ gives

$$
\begin{aligned}
\rho(\bar{\theta})-\rho(\underline{\theta}) & =\lambda+(1+\lambda) c / \bar{F}+\int_{\Theta} \gamma(\theta) d \theta \\
& =\lambda+\tau \\
& =\lambda+\rho(\bar{\theta})
\end{aligned}
$$

using (29) and (28). Consequently, using (25), $\rho(\underline{\theta})=-\lambda=\nu(\underline{\theta})$ and since

$$
\begin{aligned}
\dot{\rho}(\theta) & =(\lambda+(1+\lambda) c / \bar{F}) g(\theta)+\gamma(\theta) \\
& \geq(\lambda+(1+\lambda) c / \bar{F}) g(\theta) \\
& =\dot{\nu}(\theta)
\end{aligned}
$$

by (22), we thus have $\rho(\theta) \geq \nu(\theta)$. Moreover, since $\mu(\cdot)$ is non-increasing, if constraints (8) bind over a subset of $\Theta$, they bind over a $\left[\underline{\theta}, \theta_{1}\right]$ interval which implies $\rho(\theta)>\nu(\theta)$ for all $\theta>\underline{\theta}$. In that case, whenever $e^{*}(\theta)<q^{\circ}(\theta)$, (26) gives

$$
\begin{aligned}
\partial_{q} \pi\left(e^{*}(\theta), \theta\right) & =\frac{d-\rho(\theta) / g(\theta) \partial_{\theta q} \pi\left(e^{*}(\theta), \theta\right)}{(1+\lambda)(1+c / \bar{F}) \partial_{q} \pi\left(e^{*}(\theta), \theta\right)+\gamma(\theta)} \\
& <\frac{d-\nu(\theta) / g(\theta) \partial_{\theta q} \pi\left(e^{*}(\theta), \theta\right)}{(1+\lambda)(1+c / \bar{F}) \partial_{q} \pi\left(e^{*}(\theta), \theta\right)}
\end{aligned}
$$

and thus $e^{*}(\theta)>\hat{q}(\theta)$ whenever $e^{*}(\theta)<q^{\circ}(\theta)$. 


\section{Proof of proposition 6}

With $\nu(\cdot)$ and $\tau(\cdot)$ as the multipliers for (17) and (19) respectively (with $\tau(\cdot) \geq 0$ ), the Lagrangian of program IV is given by

$$
\mathcal{L}=\int_{\theta_{0}}^{\bar{\theta}} H(\theta) d \theta-(1+\lambda) \frac{c}{\bar{F}}\left(\pi^{\circ}(\bar{\theta})-R(\bar{\theta})-k\right)-\nu(\bar{\theta}) R(\bar{\theta})+\nu\left(\theta_{0}\right) R\left(\theta_{0}\right)
$$

where

$$
\begin{aligned}
H \equiv & ((1+\lambda) \pi(q(\theta), \theta)-d q(\theta)) g(\theta)+\nu(\theta) \partial_{\theta} \pi(q(\theta), \theta) \\
& +R(\theta)\{\dot{\nu}(\theta)-\lambda g(\theta)\}+\tau(\theta)(\pi(q(\theta), \theta)-R(\theta)-k)
\end{aligned}
$$

Pointwise maximizations give

$$
\begin{aligned}
\frac{\partial H}{\partial q}= & \left((1+\lambda) \partial_{q} \pi(q(\theta), \theta)-d\right) g(\theta)+\nu(\theta) \partial_{\theta q} \pi(q(\theta), \theta) \\
& +\tau(\theta) \partial_{q} \pi(q(\theta), \theta) \geq 0\left(=0 \text { if } q(\theta)<q^{\circ}(\theta)\right) \\
\frac{\partial H}{\partial R}= & \dot{\nu}(\theta)-\lambda g(\theta)-\tau(\theta)=0
\end{aligned}
$$

First-order conditions for $k$ and $\theta_{0}$ are given by

$$
\begin{gathered}
\frac{\partial \mathcal{L}}{\partial k}=-\int_{\theta_{0}}^{\bar{\theta}} \tau(\theta) d \theta+(1+\lambda) c / \bar{F} \leq 0(=0 \text { if } k>0) \\
\frac{\partial \mathcal{L}}{\partial \theta_{0}}=-H\left(\theta_{0}\right)+\dot{\nu}\left(\theta_{0}\right) R\left(\theta_{0}\right)+\nu\left(\theta_{0}\right) \dot{R}\left(\theta_{0}\right) \leq 0\left(=0 \text { if } \theta_{0}>\underline{\theta}\right) .
\end{gathered}
$$

and transversality conditions by

$$
\frac{\partial \mathcal{L}}{\partial R(\bar{\theta})}=-\nu(\bar{\theta})+(1+\lambda) c / \bar{F}=0
$$

and

$$
\frac{\partial \mathcal{L}}{\partial R\left(\theta_{0}\right)}=\nu\left(\theta_{0}\right) \leq 0\left(=0 \text { if } R\left(\theta_{0}\right)>0\right)
$$

(i) and (ii). Since $c / \bar{F}>0$ we have, from (32), $k>0$ and $\tau(\theta)>0$ for all $\theta$ in a non-negligible subset $\Theta_{1}$ of $\Theta$. Over $\Theta_{1}$, we thus have $R(\theta)=\pi(q(\theta), \theta)-k$ which gives $F(\theta)=0$. Since $F(\cdot)$ is non-decreasing, we must have $\Theta_{1}=\left[\theta_{0}, \hat{\theta}\right]$ with $\hat{\theta}>$ 
$\theta_{0}$. Moreover, from (17), we must have $(d / d \theta) \pi(q(\theta), \theta)=\partial_{\theta} \pi(q(\theta), \theta)$ which implies $\partial_{q} \pi(q(\theta), \theta) \dot{q}(\theta)=0$ and thus either $q(\theta)=q^{\circ}(\theta)$ (we then have $\partial_{q} \pi\left(q^{\circ}(\theta), \theta\right)=0$ ) or $q(\theta)=z$ for all $\theta \leq \hat{\theta}$. Assuming $q(\theta)=q^{\circ}(\theta),(30)$ implies

$$
\nu(\theta) \partial_{\theta q} \pi\left(q^{\circ}(\theta), \theta\right)>g(\theta)>0
$$

for all $\theta \in\left[\theta_{0}, \hat{\theta}\right)$. By integrating (31) over $[\theta, \bar{\theta}]$ and using (34), we have

$$
\nu(\theta)=(1+\lambda) c / \bar{F}-\lambda(1-G(\theta))-\int_{\theta}^{\bar{\theta}} \tau(x) d x
$$

and thus, using (32),

$$
\begin{aligned}
\nu\left(\theta_{0}\right) \partial_{\theta q} \pi\left(q^{\circ}\left(\theta_{0}\right), \theta_{0}\right) & =\left((1+\lambda) c / \bar{F}-\lambda\left(1-G\left(\theta_{0}\right)\right)-\int_{\theta_{0}}^{\bar{\theta}} \tau(\theta) d \theta\right) \partial_{\theta q} \pi\left(q^{\circ}\left(\theta_{0}\right), \theta_{0}\right) \\
& =-\lambda\left(1-G\left(\theta_{0}\right)\right) \partial_{\theta q} \pi\left(q^{\circ}\left(\theta_{0}\right), \theta_{0}\right) \leq 0
\end{aligned}
$$

hence a contradiction. We thus have $q(\theta)=z<q^{\circ}\left(\theta_{0}\right)$ for all $\theta \in\left[\theta_{0}, \hat{\theta}\right]$. Using (32) and (36), $k>0$ implies $\nu\left(\theta_{0}\right)=-\lambda\left(1-G\left(\theta_{0}\right)\right)<0$ and thus $R\left(\theta_{0}\right)=\pi\left(z, \theta_{0}\right)-k=0$. (33) simplifies to

$$
-\left((1+\lambda) \pi\left(z, \theta_{0}\right)-d z\right) g\left(\theta_{0}\right) \leq 0\left(=0 \text { if } \theta_{0}>\underline{\theta}\right)
$$

and we have $\theta_{0}>\underline{\theta}$ if $(1+\lambda) \pi(z, \underline{\theta})-d z>0$.

(iii). Plugging (31) into (30) gives, for all $\theta \in\left[\theta_{0}, \hat{\theta}\right)$,

$$
\dot{\nu}(\theta)+\nu(\theta) \partial_{\theta q} \pi(z, \theta) / \partial_{q} \pi(z, \theta)+\left(1-d / \partial_{q} \pi(z, \theta)\right) g(\theta)=0
$$

which is a linear first-order differential equation in $\nu(\cdot)$. The solution is given by $\nu(\theta)=h(\theta) r(\theta)$ where

$$
\begin{aligned}
\dot{r}(\theta)+r(\theta) \partial_{\theta q} \pi(z, \theta) / \partial_{q} \pi(z, \theta) & =0 \\
\dot{h}(\theta) r(\theta)+\left(1-d / \partial_{q} \pi(z, \theta)\right) g(\theta) & =0
\end{aligned}
$$

which gives $r(\theta)=1 / \partial_{q} \pi(z, \theta)$ and

$$
h(\theta)=h\left(\theta_{0}\right)+d\left[G(\theta)-G\left(\theta_{0}\right)\right]-\int_{\theta_{0}}^{\theta} \partial_{q} \pi(z, x) g(x) d x
$$


From $\nu\left(\theta_{0}\right)=-\lambda\left(1-G\left(\theta_{0}\right)\right)$ we have

$$
\nu(\theta)=-\lambda\left(1-G\left(\theta_{0}\right)\right) \frac{\partial_{q} \pi\left(z, \theta_{0}\right)}{\partial_{q} \pi(z, \theta)}+d \frac{G(\theta)-G\left(\theta_{0}\right)}{\partial_{q} \pi(z, \theta)}-\int_{\theta_{0}}^{\theta} \frac{\partial_{q} \pi(z, x)}{\partial_{q} \pi(z, \theta)} g(x) d x
$$

for all $\theta<\hat{\theta}$. By continuity of $\nu(\cdot)$, we have $\nu\left(\hat{\theta}^{-}\right)=\nu\left(\hat{\theta}^{+}\right)$hence

$-\lambda\left(1-G\left(\theta_{0}\right)\right) \frac{\partial_{q} \pi\left(z, \theta_{0}\right)}{\partial_{q} \pi(z, \hat{\theta})}+d \frac{G(\hat{\theta})-G\left(\theta_{0}\right)}{\partial_{q} \pi(z, \hat{\theta})}-\int_{\theta_{0}}^{\hat{\theta}} \frac{\partial_{q} \pi(z, x)}{\partial_{q} \pi(z, \hat{\theta})} g(x) d x=(1+\lambda) \frac{c}{\bar{F}}-\lambda(1-G(\hat{\theta}))$

From (30) and (36), we also obtain

$$
\left((1+\lambda) \partial_{q} \pi(z, \hat{\theta})-d\right) g(\hat{\theta})+((1+\lambda) c / \bar{F}-\lambda(1-G(\hat{\theta}))) \partial_{\theta q} \pi(z, \hat{\theta})=0
$$

(iv) For all $\theta>\hat{\theta},(36)$ gives

$$
\nu(\theta)=(1+\lambda) c / \bar{F}-\lambda(1-G(\theta))
$$

Plugging this expression into (30) gives (20). 